\title{
Cardiac Computed Tomography Assessment in Acute Coronary Syndromes - Do We Have Time for It in Emergency Settings?
}

\author{
Alexandra Stănescu1,2, Elisabeta Himcinschi², Mirabela Morariu11,2, Nora Rat'1,2, Lehel Bordi², \\ Mihaela Rațiu'1,2, Annabella Benedek ${ }^{1}$ Imre Benedek ${ }^{1,2}$, Theodora Benedek ${ }^{1,2}$ \\ 1 University of Medicine and Pharmacy, Tîrgu Mureș, Romania \\ 2 Center of Advanced Research in Multimodality Cardiac Imaging, Cardio Med Medical Center, Tîrgu Mureș, Romania
}

\section{CORRESPONDENCE}

\section{Elisabeta Himcinschi}

Str. 22 Decembrie 1989 nr. 76

540124 Tîrgu Mureș, Romania

Tel: +40 265217333

E-mail: eli_himcinschi@yahoo.com

\section{ARTICLE HISTORY}

Received: 13 September, 2016 Accepted: 14 November, 2016

Alexandra Stănescu • Str. Gheorghe Marinescu n 38, 540139 Tîrgu Mureș, Romania. Tel: +40 265215 551. E-mail: alexandrastanescu90@gmail.com

Mirabela Morariu • Str. Gheorghe Marinescu nr. 38 540139 Tîrgu Mureș, Romania. Tel: +40 265215551. E-mail: mirabela.morariu@yahoo.com

Nora Rat • Str. Gheorghe Marinescu nr. 38, 540139 Tîrgu Mureș, Romania. Tel: +40 265215 551. E-mail: ratnora@gmail.com

Lehel Bordi • Str. 22 Decembrie 1989 nr. 76, 540124 Tîrgu Mureș, Romania. Tel: +40 265217 333. E-mail: bordi_lehel@yahoo.com

Mihaela Ratiu • Str. 22 Decembrie 1989 nr. 76 540124 Tîrgu Mures, Romania. Tel: +40 265217333. E-mail: d_a mihaela@yahoo.com

Annabella Benedek • Str. Gheorghe Marinescu nr. 38 540139 Tîrgu Mureș, Romania. Tel: +40 265215551. E-mail: annabell.benedek@yahoo.com

Imre Benedek • Str. Gheorghe Marinescu nr. 38 540139 Tîrgu Mureș, Romania. Tel: +40 265215551. E-mail: imrebenedek@yahoo.com

Theodora Benedek • Str. Gheorghe Marinescu nr. 38 540139 Tîrgu Mures, Romania. Tel: +40 265215551 .

E-mail: theodora.benedek@gmail.com

\begin{abstract}
The diagnosis and treatment of acute coronary syndrome remain a challenge for clinicians in many clinical settings, especially in patients with previous low-to-intermediate risk. Due to its high specificity and sensitivity for detecting significant coronary artery stenoses, cardiac computed tomography angiography (CCTA) tends to be used more frequently in the emergency room (ER) in the last years. This technique has been associated with a higher rate of safe discharge in patients with chest pain, less time spent in the ER, and decreased costs related to further investigations. In cases positive for coronary artery stenosis, CCTA can accurately evaluate the indication for percutaneous coronary angioplasty and can offer relevant information related to the characteristics of the coronary plaques, being able to detect vulnerable coronary plaques. The aim of this manuscript is to highlight the possibility of using CCTA in the ER in the assessment of patients with chest pain and to show the benefits of the procedure regarding safety, costs, accuracy, and time.
\end{abstract}

Keywords: coronary computed tomography angiography, acute coronary syndrome, emergency department

\section{INTRODUCTION}

Coronary computed tomography angiography (CCTA) became a widely used imagistic technique, due to its high specificity and accuracy for detecting coronary artery diseases $(\mathrm{CAD})$. At the same time, this technique provides relevant data regarding coronary anatomy, calcium scoring, coronary lumen and coronary plaque characteristics, via a noninvasive route. ${ }^{1}$ CCTA is frequently used in the emergency room (ER), being a reliable procedure that allows the clinician to safely discharge the patients with suspected acute coronary syndrome and no coronary artery stenosis. ${ }^{2,3}$ 
Despite of many developments in the diagnosis and treatment of this devastating disease, acute coronary syndrome (ACS) remains a major cause of mortality worldwide. ${ }^{4}$ This pathology includes acute myocardial infarction and unstable angina. According to data published by the American Heart Association, approximately 1.5 million patients were discharged in 2005 in the USA with ACS. ${ }^{5}$ Recent data regarding ACS shows that 7 million deaths occur annually due to ischemic heart diseases and a number of 129 million patients present loss of disability-adjusted life years (DALYs) annually worldwide after a coronary ischemic event. ${ }^{6,7}$

In patients presenting to the ER with acute chest pain and evaluated at low-to-intermediate risk for ACS, CCTA can offer relevant details regarding the pa-tient's condition. ${ }^{8}$ A negative scan could help the physician to safely discharge the patients who were admitted to the ER for chest pain, without exposing the patient to an unnecessary invasive evaluation. Available data has shown that CCTA has also a high predictive value for favorable outcomes at 1-year follow-up. ${ }^{9}$ According to several studies, CCTA is associated with a sensitivity and specificity ranging from $91 \%$ to $99 \%$ and $74 \%$ to $96 \%$, respectively. ${ }^{10-14}$ ACCURACY was an important clinical study that showed a great performance of CCTA in evaluating coronary stenosis in patients with coronary artery diseases and stable angina. ${ }^{15}$ Another multicenter study including 360 patients with acute stable and unstable angina found a similar percentage for the sensitivity and specificity of this imagistic procedure, reported as $99 \%$ and $64 \%$ respectively. ${ }^{16}$

Many published studies have shown that CCTA can offer an accurate diagnosis and emphasized its high specificity and sensitivity. However, almost all of these studies describe the utility of CCTA in patients with stable angina. In this article, we aim to present the benefits of CCTA for patients presenting to the ER, in emergency settings.

\section{CCTA IN THE EVALUATION OF PATIENTS WITH ACS WITHOUT ST-SEGMENT ELEVATION}

Due to the lack of effective protocols for ACS without ST-segment elevation in patients at low-to-intermediate risk, CCTA could be an integrated procedure the patients presenting at the ER for unstable angina. For instance, Meijboom et al. studied this fact on 104 patients with ACS without ST-segment elevation, in whom CCTA was performed, followed by traditional coronary angiography. Compared to invasive coronary angiography, CCTA seemed to have a higher sensitivity (reported as 100\%) for the diagnosis of significant coronary stenosis. ${ }^{17}$
Hoffmann et al. reported similar results in their study on 103 patients pre-senting to the ER for acute chest pain. They also evaluated the time spent for the entire procedure, noting that preparation of the patient, scan time, and interpretation time can take almost 35 minutes. Overall, 14 of the patients had ACS, while for the rest of the patients ACS was ruled out by CCTA, the patients being safely discharged. Five of the patients had myocardial infarction and they underwent PCI immediately. The authors found a good negative predictability of the procedure. ${ }^{18}$

Another study that has shown the high sensitivity of CCTA was the ROMICAT study, which involved 368 patients with chest pain and low-to-intermediate risk for ACS. Half of them did not have any coronary lesions, and CCTA helped in reducing the time spent for further investigations and the cost of unnecessary hospitalization. Nineteen percent of the patients were evaluated according to CCTA characteristics as having significant coronary artery stenosis. At the 2-year follow-up the researchers found that $6.8 \%$ of the patients had acute myocardial infarction or needed coronary revascularizations. At the same time, CCTA demonstrated a high prognostic value in detecting the probability of major cardiac events in the follow-up period. ${ }^{19}$

Motoyama et al. evaluated the CCTA characteristics of lesions causing ACS. They examined 38 patients with ACS and compared them with 33 patients diagnosed with stable angina pectoris. They found that positive vascular remodeling, plaque density $<30 \mathrm{HU}$, and spotty calcifications were characteristic for plaques associated with acute ischemic events. ${ }^{20}$

\section{BENEFITS OF CCTA IN ACUTE SETTINGS}

CCTA could represent a reliable diagnostic procedure in patients with ACS admitted to the ER, who were recommended in the past to perform a stress test. Today, it is considered that CCTA could replace the traditional stress tests that are sometimes difficult to be interpreted. ${ }^{17}$

However, we should remember that all patients who are admitted to the ER for chest pain and present an STsegment elevation on surface ECG should undergo PCI as soon as possible. In these cases, performing a CCTA can be time-consuming and should be avoided, as time is critical for the outcome of the patient. ${ }^{21}$ CCTA can rule out multivessel disease or unsuitable anatomy for PCI that requires coronary artery bypass surgery (CABG) in the acute phase of STEMI. An urgent indication for CABG could be represented by the cardiogenic shock associated with unsuitable anatomy not amendable for PCI, as identified by CCTA. 
These are still a few patients that were described in the literature. $^{22}$

Litt et al. published a multicenter study in which they enrolled 1,392 patients with suspected ACS who were admitted to the ER for acute chest pain. ${ }^{3}$ They randomized the patients into 2 groups, the first group consisting of patients who underwent CCTA, and the second group consisting of patients investigated with traditional protocols. They compared the two groups and found that patients who underwent CCTA were discharged from the ER in less time than patients from the traditional care group. Moreover, coronary artery disease was diagnosed in a high percentage in the CCTA group compared to the second group: $9.9 \%$ vs. $3.5 \%$. They concluded that CCTA can be safely included in the protocols of the ER for suspected acute coronary syndromes in patients at low-to-intermediate risk. ${ }^{3}$

Hulten et al. performed a systematic meta-analysis evaluating the accuracy of CCTA in acute settings, in the ER, for patients with suspected ACS. They included in their analysis studies that compared the outcome of patients evaluated with CCTA and usual care. ${ }^{3,23-25}$ The authors observed that compared to usual care, patients who performed CCTA required less costs and decreased length of stay in the hospital. Moreover, the CCTA group consisted of patients who required PCI and revascularization more than the usual care group ( $4.6 \%$ vs. $2.6 \%)$. This was explained by the fact that usual care cannot always rule out CAD and it was misdiagnosed. ${ }^{26}$

According to the existing recommendations, in order to obtain high quality images, patients should be prepared in the ER according to standard CCTA protocols, in order to obtain a stable heart rate, which can prolong the procedure-related time in certain cases. ${ }^{27}$

\section{CONCLUSION}

CCTA can be used in emergency settings for the evaluation of patients with acute coronary syndrome without ST-segment elevation, assessed as having a low-to-intermediate risk. This procedure can reduce the time spent with diagnosis in the ER, in parallel with a significant reduction of the costs associated with additional investigations. CCTA has a high accuracy in detecting significant coronary stenosis and can help to establish the best therapeutic choice in patients with suspected acute coronary syndromes presenting to the ER in acute settings.

\section{CONFLICT OF INTEREST}

Nothing to declare.

\section{ACKNOWLEDGEMENT}

This research was supported via the research grant no. 103545/2016, contract number 43/05.09.2016, entitled "High performance multimodal MRI/CT imagingplatform, for applications in computational medicine, nanoparticles and hybrid imaging for the research of atherothrombotic disorders - CARDIO IMAGE" financed by the Romanian Ministry of European Funds, the Romanian Government and the European Union.

\section{REFERENCES}

1. Prazeres CE, Cury RC, Carneiro AC, Rochitte CE. Coronary Computed Tomography Angiography in the Assessment of Acute Chest Pain in the Emergency Room. Arquivos Brasileiros de Cardiologia. 2013;101:562-569.

2. Sato $Y$, Imazeki $T$, Inoue $F$, et al. Detection of atherosclerotic coronary artery plaques by multislice computed tomography in patients with acute coronary syndrome: Report of 2 cases. Circ J. 2004;68:263-266.

3. Litt H, Gatsonis C, Snyder B, et al. CT angiography for safe discharge of patients with possible acute coronary syndromes. $N$ Engl J Med. 2012;366:1393-1403.

4. Boden WE, Shah PK, Gupta V, Ohman EM. Contemporary approach to the diagnosis and management of non-ST-segment elevation acute coronary syndromes. Prog Cardiovasc Dis. 2008;50:311-351.

5. Rosamond W, Flegal K, Furie K, et al. Heart disease and stroke statistics-2008 update: a report from the American Heart Association Statistics Committee and Stroke Statistics Subcommittee. Circulation. 2008;117(4):e25-e146

6. Lozano R, Naghavi M, Foreman K, et al. Global and regional mortality from 235 causes of death for 20 age groups in 1990 and 2010: a systematic analysis for the Global Burden of Disease Study 2010. Lancet. 2012;380:2095-2128

7. Murray CJ, Vos T, Lozano R, et al. Disability-adjusted life years (DALYS) for 291 diseases and injuries in 21 regions, 1990-2010: a systematic analysis for the Global Burden of Disease Study 2010. Lancet. 2012;380:21972223.

8. Prazeres CE, Cury RC, Carneiro AC, Rochitte CE. Coronary computed tomography angiography in the assessment of acute chest pain in the emergency room. Arquivos brasileiros de cardiologia. 2013;101:562-569.

9. Gilard M, Le Gal G, Cornily JC, et al. Midterm prognosis of patients with suspected coronary artery disease and normal multislice computed tomographic findings: a prospective management outcome study. Arch Intern Med. 2007;167:1686-1689

10. Abdulla J, Abildstrom SZ, Gotzsche O, Christensen E, Kober L, TorpPedersen C. 64-multislice detector computed tomography coronary angiography as potential alternative to conventional coronary angiography: a systematic review and meta-analysis. Eur Heart J. 2007;28:3042-3050.

11. Mowatt G, Cook JA, Hillis GS, et al. 64-Slice computed tomography angiography in the diagnosis and assessment of coronary artery disease: systematic review and meta-analysis. Heart. 2008;94:1386-1393.

12. Mowatt G, Cummins E, Waugh N, et al. Systematic review of the clinical effectiveness and cost-effectiveness of 64-slice or higher computed tomography angiography as an alternative to invasive coronary angiography in the investigation of coronary artery disease. Health Technol Assess. 2008;12:iii-iv,ix-143.

13. Hamon M, Biondi-Zoccai GG, Malagutti P, et al. Diagnostic performance of multislice spiral computed tomography of coronary arteries as compared with conventional invasive coronary angiography: a meta-analysis. J Am Coll Cardiol. 2006;48:1896-1910.

14. Hamon M, Morello R, Riddell JW. Coronary arteries: diagnostic performance of 16- versus 64-section spiral CT compared with invasive coronary angiography-meta-analysis. Radiology. 2007;245:720-731.

15. Budoff MJ, Dowe D, Jollis JG, et al. Diagnostic performance of 64-multidetector row coronary computed tomographic angiography for evaluation of coronary artery stenosis in individuals without known coronary artery disease: results from the prospective multicenter ACCURACY (Assessment by Coronary Computed Tomographic 
Angiography of Individuals Undergoing Invasive Coronary Angiography) trial. J Am Coll Cardiol. 2008;52:1724-1732.

16. Meijboom WB, Meijs MF, Schuijf JD, et al. Diagnostic accuracy of 64-slice computed tomography coronary angiography: a prospective, multicenter multivendor study. J Am Coll Cardiol. 2008;52:2135-2144.

17. Meijboom WB, Mollet NR, Van Mieghem CA, et al. 64-Slice CT coronary angiography in patients with non-ST elevation acute coronary syndrome. Heart. 2007;93:1386-1392.

18. Hoffmann U, Nagurney JT, Moselewski F, et al. Coronary multidetector computed tomography in the assessment of patients with acute chest pain. Circulation. 2006:114:2251-2260.

19. Hoffmann U, Bamberg F, Chae CU, et al. Coronary computed tomography angiography for early triage of patients with acute chest pain: the ROMICAT (Rule Out Myocardial Infarction using Computer Assisted Tomography) trial. J Am Coll Cardiol. 2009;53:1642-1650.

20. Motoyama S, Kondo T, Sarai M, et al. Multislice computed tomographic characteristics of coronary lesions in acute coronary syndromes. J Am Coll Cardiol. 2007;50:319-326.

21. Kornowski R, Mehran R, Dangas G, et al. Prognostic impact of staged versus "one-time" multivessel percutaneous interventions in acute myocardial infarction: analysis from the HORIZONS-AMI trial. J Am Coll Cardiol. 2011;58:704-711.
22. Van de Werf F, Bax J, Betriu A et al. Management of acute myocardial infarction in patients presenting with persistent ST-segment elevation: the task force on the management of ST-segment elevation acute myocardial infarction of the European Society of Cardiology. Eur Heart J. 2008;29:2909-2945

23. Goldstein JA, Gallagher MJ, O'Neill WW, Ross MA, O'Neil BJ, Raff GL. A randomized controlled trial of multislice coronary computed tomography for evaluation of acute chest pain. J Am Coll Cardiol. 2007;49:863-871.

24. Goldstein JA, Chinnaiyan KM. Abidov A, et al. The CT-STAT (Coronary Computed Tomographic Angiography for Systematic Triage of Acute Chest Pain Patients to Treatment) trial. J Am Coll Cardiol. 2011;58:14141422.

25. Hoffmann U, Truong QA, Schoenfeld DA, et al. Coronary CT angiography versus standard evaluation in acute chest pain. N Engl $J$ Med. 2012;367:299-308.

26. Hulten E, Pickett C, Bittencourt MS, et al. Outcomes after coronary computed tomography angiography in the emergency department: a systematic review and meta-analysis of randomized, controlled trials. $J$ Am Coll Cardiol. 2013;61:880-892.

27. Frauenfelder $\mathrm{T}$, Appenzeller $\mathrm{P}$, Karlo $\mathrm{C}$, et al. Triple rule-out $\mathrm{CT}$ in the emer-gency department: protocols and spectrum of imaging findings. Eur Radiol. 2009;19:789-799. 